Results We observed 2,726 pregnancy losses in a cohort of 68,096 pregnant women. We found no consistent associations between increased heavy lifting and pregnancy losses up to gestational week 21. However, for fetal death occurring after week 21 we found an increased risk among women lifting more than $200 \mathrm{~kg} /$ day (Hazard Ratio $(\mathrm{HR})=1.55 ; 95 \%$ Confidence Interval (CI): 1.03-2.35) compared to non-lifting women. After controlling for potential confounders, the result for stillbirths became insignificant, but the estimate did not change much (HR $=1.40$; 95\% CI: 0.92-2.14).

Conclusion The study corroborates earlier findings in the Danish National Birth Cohort that occupational lifting is related to an increased risk for fetal death. Due to the prospective design with late enrollment into the study, ascertainment of early miscarriage is incomplete and therefore the study does not provide strong evidence on risk for early miscarriage.

\section{PRETERM BIRTH IN RELATION TO OCCUPATIONAL LIFTING ASSESSED BY A JOB EXPOSURE MATRIX: FINDINGS FROM THE DANISH NATIONAL BIRTH COHORT}

${ }^{1} \mathrm{~S}$ W Svendsen, ${ }^{2}$ Frost, ${ }^{3}$ Mocevic, ${ }^{3}$ Jorgensen, ${ }^{4}$ Nybo Andersen, ${ }^{3}$ Bonde. ${ }^{1}$ Herning Regional Hospital, Herning, Denmark; ${ }^{2}$ Department of Occupational Medicine, Aarhus, Denmark; ${ }^{3}$ Bispebjerg University Hospital, Copenhagen, Denmark; ${ }^{4}$ University of Copenhagen, Copenhagen, Denmark

\subsection{6/oemed-2013-101717.215}

Objective The question remains unresolved if the risk of preterm birth increases with increasing occupational lifting. Previous studies have generally used individual-based exposure assessment relying on self-report, and based on this approach, we have observed an exposure-response relation between total load lifted per day and preterm birth. ${ }^{1}$ To corroborate our finding, we aimed to examine the risk of preterm birth among primigravidas in relation to occupational lifting during pregnancy using groupbased exposure assessment.

Methods Within the Danish National Birth Cohort (DNBC), we identified 24,833 occupationally active primigravidas with singleton pregnancies, who as a minimum entered gestational week 23 and provided interview data while pregnant. We constructed a Job Exposure Matrix (JEM) based on information from all women in the DNBC, who were pregnant when interviewed. The JEM cross-tabulated job and industry information with average total loads lifted per day. Each woman received an exposure estimate from the JEM. We used Cox regression analysis adjusting for age, smoking, BMI, and alcohol consumption. The women were followed from start of week 23 or interview date, whichever came last, until end of week 37 or pregnancy termination, whichever came first.

Results A total of 1601 preterm births occurred. Adjusted HRs increased with increasing occupational lifting, reaching a HR of 1.42 (95\% CI 1.13-1.77) for women in the highest exposure category (>200 kg per day), when compared to non-lifters.

Conclusion We used group-based exposure assessment to minimise information bias and attenuation of exposure-response relations. Among women in jobs categorised with a lifting exposure of $>200 \mathrm{~kg}$ per day, we found support for a moderately increased risk of preterm birth.

\section{REFERENCE}

1 Runge SB, Pedersen JK, Svendsen SW, Juhl M, Bonde JP, Nybo Andersen AM. Occupational lifting and preterm birth: a study within the Danish National Birth Cohort. EPICOH 2011 (abstract).
Session: Parallel session 1 RICOH: Stress, shift work and occupations with chemical exposure

\section{RISK OF CRYPTORCHIDISM IN SONS OF FARMERS AND HORTICULTURAL WORKERS IN DENMARK}

${ }^{1} \mathrm{~K}$ T Jørgensen, ${ }^{2}$ Jensen, ${ }^{2} \mathrm{Toft},{ }^{3}$ Larsen, ${ }^{1}$ Bonde, ${ }^{3}$ Hougaard. 'Bispebjerg Hospital, Copenhagen NV, Denmark; ${ }^{2}$ Aarhus University Hospital, Aarhus, Denmark; ${ }^{3}$ The National Research Centre for the Working Environment, Copenhagen, Denmark

\subsection{6/oemed-2013-101717.216}

Objectives Previous studies indicate that sons of women working with pesticides may have an increased risk of cryptorchidism. This study assessed the risk of cryptorchidism among boys of parents employed as farmers or horticultural workers using nationwide registers on parental occupation and cryptorchidism diagnoses.

Methods Our study cohort of more than 600,000 boys included all boys born in Denmark from 1980 to 2007 with a mother or father in employment during pregnancy. The cohort was followed for the occurrence of cryptorchidism and orchiopexy from 1980 to 2009 comparing the risk in sons of horticultural workers and farmers with sons of parents in other occupations. Hazard ratios (HR) and 95\% confidence intervals (CI) were estimated using Cox regression adjusting for maternal and paternal age, birth years and parity.

Results Maternal employment as farmer was associated with moderately increased risks of cryptorchidism (cases 157; HR 1.31; 95\% CI 1.12-1.53) and orchiopexy (cases 111; HR 1.29; 95\% CI 1.07-1.56) compared to children of mothers in other occupations (15511 cases of cryptorchidism and 9963 of orchiopexy). Paternal occupation as farmer was unrelated to the risk in sons. Maternal occupation as horticultural worker was associated with non-significantly increased risks of cryptorchidism (cases 72; HR 1.20; 95\% CI 0.95-1.52) and orchiopexy (cases 51; HR $1.28 ; 0.97-1.68)$. Similar associations were found for paternal horticultural workers. Prior but not current maternal employment as horticultural worker or farmer was not associated with an increased risk.

Conclusions This register-based study provides support for a possible association between maternal employment as a farmer during pregnancy and cryptorchidism in boys. Our finding of similarly increased risks in sons of mothers and fathers employed as horticultural workers question whether this association is causally related to pesticide exposure or has alternative explanations.

\section{PREGNANCIES IN A PROSPECTIVE COHORT OF WOMEN IN NON-TRADITIONAL WORK (THE WHAT-ME STUDY)}

M Nicola, Arrandale, Beach. University of Alberta, Edmonton, Canada

\subsection{6/oemed-2013-101717.217}

Objectives The WHAT-ME study (Women's health in apprenticeship trades- metalworkers and electricians) was established because of concerns about risk to the fetus of women welding in pregnancy.

Methods Women in registered apprenticeships since 2005 in welding, boiler-making, steam fitting/pipefitting ('welders') or electrical trades are invited to join the study. They complete questionnaires on health and exposure at baseline and 